\title{
Las empresas forestales comunitarias en la región de la Mariposa Monarca, México: un enfoque empresarial
}

\author{
The community forestry enterprise in Monarch Butterfly Region, Mexico: \\ a business approach
}

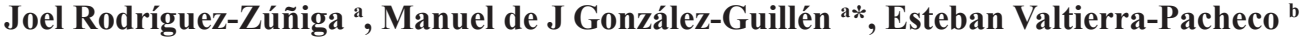

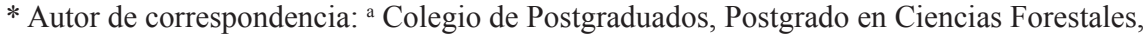 \\ Carretera México-Texcoco km 36,5, Montecillo, Texcoco, Estado de México, México, CP. 56230, tel.: (52-595)9520246, \\ manuelg@colpos.mx \\ ${ }^{\mathrm{b}}$ Colegio de Postgraduados, Postgrado en Desarrollo Rural, Carretera México-Texcoco km 36,5, Montecillo, \\ Texcoco, Estado de México, México.
}

\begin{abstract}
SUMMARY
The absence of a consensus in the management of a community forestry enterprise can lead to inadequate management, inefficient use of resources and low competitiveness. To reverse this situation, it is important that people work in groups cooperating each other and achieving common goals, based on a set of shared informal norms and values called corporate principles. The objective of this research was to analyze the corporate principles of the forest communal enterprises in the Monarch Butterfly Biosphere Reservoir and their relation to the integration of the forest production chain and the conservation of natural resources. This research used structured and semi-structured surveys, a spatial analysis in a digital platform, and construction of a development index based on multivariate methods. Three enterprises with different levels of integration of the forest production chain were compared. Main findings showed that when the community enterprise integrates more segments into a forest production chain, members have better knowledge of the corporative principles. Forest community enterprises with higher productive integration have higher levels of conservation of their forestry, more economic benefits for their members and generate more social welfare for their community. It is recommended that public policies focused on forest communities should foster corporative culture to integrate adequately production chains of forest community enterprises.
\end{abstract}

Key words: strategic analysis, production chain, forest community, corporative principles, social capital.

\section{RESUMEN}

La ausencia de un consenso en el manejo de una empresa forestal comunitaria puede conducir a una administración inadecuada, a un uso ineficiente de recursos y a una competitividad baja. Para revertir esta situación, es importante que las personas trabajen en grupo, para cooperar entre sí y poder lograr objetivos comunes, sobre la base de un conjunto de normas y valores informales compartidos denominados principios corporativos. El objetivo de esta investigación fue analizar los principios corporativos de las empresas forestales comunitarias de la Reserva de la Biosfera de la Mariposa Monarca y su relación con la integración de la cadena productiva forestal y la conservación de los recursos naturales. La investigación utilizó la encuesta estructurada y semiestructurada; el análisis espacial se realizó en una plataforma digital y la construcción de índices de desarrollo mediante métodos multivariados. Se compararon tres empresas que tenían diferentes niveles de integración de la cadena productiva. Los resultados indicaron que en la medida que las empresas forestales comunitarias integran más eslabones a la cadena productiva, los miembros tienen mayor conocimiento de los principios corporativos. Las empresas forestales comunitarias de la Reserva que tienen mayor integración productiva presentaron mayores niveles de conservación de sus bosques, mayores beneficios económicos para sus miembros y un mayor bienestar social para su comunidad. Se recomienda que las políticas públicas dirigidas a las comunidades forestales fomenten la cultura corporativa para integrar adecuadamente las cadenas productivas de las empresas forestales comunitarias.

Palabras clave: análisis estratégico, cadena productiva, comunidad forestal, principios corporativos, capital social.

\section{INTRODUCCIÓN}

En México, las empresas forestales comunitarias (EFC) tienen su origen en el ejido o en la comunidad agraria; estas últimas son organizaciones sociales dueñas de terrenos forestales de forma comunal que poseen diversos niveles de integración productiva y que producen madera con fines comerciales. Desde la perspectiva administrativa y empresarial, muchas de las empresas forestales comunitarias mexicanas no han sido exitosas debido a la impo- 
sición de programas gubernamentales de conservación y modelos inadecuados para integrarse a una economía de mercado (Bray 2007). De un total de 584 comunidades forestales que existen en México, son pocas las que se han constituido como empresas forestales comunitarias competitivas que tienen una visión empresarial. Algunos ejemplos sobresalientes de empresas forestales comunitarias en México incluyen: las comunidades agrarias de Ixtlán de Juárez y San Pedro el Alto en el estado de Oaxaca; San Juan Nuevo en Michoacán; el ejido El Balcón en Guerrero, el ejido Pueblo Nuevo en Durango y el ejido El Largo en el estado de Chihuahua (Garibay 2005).

Las empresas forestales comunitarias se rigen por principios éticos que buscan satisfacer las necesidades sociales y medioambientales de sus integrantes y del entorno natural (Maignan y Ferrell 2004). Estas obtienen ganancias a través de su participación en el mercado de bienes y para ello requieren competitividad y una cultura organizacional para poder sobrevivir. La organización de una empresa se basa en fines comunes de sus miembros con valores, creencias, normas y una visión empresarial (Porter 1991). La ausencia de un consenso comunitario en el manejo de los bosques de una empresa forestal comunitaria puede conducir a una administración inadecuada, a un uso ineficiente de recursos y a una competitividad baja. De tal manera, que un elemento fundamental en el manejo de las empresas forestales comunitarias es conocer la percepción de los miembros de la comunidad sobre el modelo de aprovechamiento de los recursos que desean. Esto permitiría maximizar los objetivos sociales realizando un manejo adecuado y competitivo bajo una economía de mercado.

La Reserva de la Biosfera Mariposa Monarca (RBMM) es el hábitat natural de destino final de la Mariposa Monarca (Danaus plexippus L.) que migra desde Canadá. En 1980 fue decretada Zona de Reserva y Refugio de Fauna Silvestre con lo que inició una serie de acciones contrastantes en el manejo de los recursos forestales. Por muchos años, el Estado mexicano excluyó casi completamente a las comunidades forestales para participar en la definición de políticas públicas dirigidas al manejo de los recursos forestales. La mayor parte de las iniciativas gubernamentales fueron impuestas a las comunidades, con poca o ninguna consulta previa a sus integrantes. En 1986, el Estado inicialmente declaró 16.110 ha (en la actualidad tiene una extensión de 56.259 ha) como Reserva de la Biosfera de la Mariposa Monarca considerando que sería la mejor opción para el bienestar de sus moradores y para la conservación de los bosques donde hiberna la mariposa monarca. En estos procesos, el Estado no permitió la autogestión de los ejidos y comunidades haciéndolas dependientes de decisiones externas e impidiéndoles una participación efectiva en el manejo de sus recursos forestales (PEF 2001).

El resultado de varias décadas de programas gubernamentales erróneos afectó drásticamente la economía de las comunidades y sus bosques. El culmen de estas políticas erróneas fue la declaratoria de la Reserva de la Biosfera de la Mariposa Monarca en 1986 y la implementación de varios proyectos productivos que pretendían fomentar la producción forestal sustentable; sin embargo, fracasaron rotundamente y, más aún, la tala ilegal de los bosques se agudizó a partir de la declaratoria (Bray 2007).

La tendencia del gobierno a forzar a las comunidades rurales hacia modelos de "desarrollo" sin considerar la opinión y las expectativas de sus integrantes, ha llevado al fracaso las políticas y proyectos de inversión gubernamental. Ejemplos de proyectos fallidos de políticas públicas en México incluyen al modelo Chontalpa en el estado sureño de Tabasco y el Programa de Desarrollo Rural Integral del Trópico Húmedo que se implementó en muchas regiones del trópico seco y trópico húmedo del país (Murillo 2004).

En México, los estudios sobre el aprovechamiento de los recursos forestales comunitarios se han centrado mayoritariamente en la discusión del funcionamiento de las comunidades forestales, sus procesos organizacionales, en la autogestión y la gobernanza de los recursos naturales comunes. En ellos, se ha revivido la discusión de la famosa disyuntiva planteada por Hardin (1968) sobre la "Tragedia de los Comunes" y que, décadas después, Ostrom (2000) criticó objetivamente ofreciendo alternativas basadas en la gobernanza de recursos naturales de uso común y la construcción de instituciones comunitarias fuertes (Stonehouse y Snowdon 2007). El capital social, por tanto, es determinante para definir los criterios de manejo y organización de la producción forestal, la gobernanza y el desarrollo económico (López et al. 2010) los cuales promueven un buen manejo del capital natural y de organizaciones con responsabilidad social. El capital social de una organización o empresa es la capacidad de las personas de trabajar en grupo, para cooperar entre sí y poder lograr determinados objetivos comunes, sobre la base de un conjunto de normas y valores informales compartidos (Fukuyama 2003); en este estudio estos aspectos se identifican como principios corporativos.

Pocos trabajos han analizado a las empresas forestales comunitarias bajo la teoría de la organización empresarial industrial (Antinori 2007), la cual ofrece un marco apropiado para analizar a las organizaciones comunitarias debido a su enfoque hacia la comparación de los beneficios de las distintas opciones de integración de una cadena de producción forestal. Algunos ejemplos de esta visión incluyen el análisis de los costos de transacción y la teoría de los contratos (Antinori 2007), los determinantes de éxito económico de una organización y el análisis estratégico (Torres-Rojo et al. 2007), y los principios corporativos (Garibay 2005).

El objetivo de esta investigación es analizar los principios corporativos de las empresas forestales comunitarias comparando la empresa de Angangueo (estado de Michoacán), que tiene un menor grado de desarrollo de la cadena productiva, con las empresas de San Juan Xoconusco (estado de Michoacán) y Agua Bendita (estado de México), bajo la hipótesis de que estas dos últimas evolucionaron 
hacia una visión empresarial en congruencia con objetivos de maximizar los beneficios sociales y conservar sus recursos forestales.

\section{MÉTODOS}

Área de estudio. La empresa forestal comunitaria (EFC) de Angangueo (figura 1) cuenta con 310 ejidatarios, tiene una superficie total de 4.196 ha de las cuales, $69 \%$ se ubica dentro de la Reserva de la Biosfera de la Mariposa Monarca. La EFC en el ANP de la Monarca se puede clasificar por el tipo del proceso productivo forestal o cadena productiva forestal (CPF) en: (1) EFC con Producción (producción de planta, mantenimiento y protección) y Abastecimiento; (2) EFC con Producción, Abastecimiento y Transformación; y (3) EFC con Producción, Abastecimiento, Transformación y Comercialización (Rodríguez et al. 2018). La EFC de Angangueo tiene el modelo (1) y es el más común en la región del Área Natural Protegida. No cuenta con aserradero u otras formas de industria y está integrada a cadenas comerciales fuera de su control.

La empresa forestal comunitaria de San Juan Xoconusco (figura 1) está integrada por 211 ejidatarios quienes han reinvertido parte de sus ganancias para instalar y equipar un aserradero, con apoyo de subsidios y créditos gubernamentales. Esto les permite procesar su materia prima para transformarla en productos escuadrados (e.g., tablas, tablones, polines, vigas, otros). La empresa abarca una superficie de 1.652 ha, de las cuales más del $90 \%$ es bosque y aproximadamente $97 \%$ se ubica en la Reserva de la Mariposa Monarca. El modelo de cadena productiva de esta empresa incluye la producción (producción de planta, mantenimiento y protección), corte y abastecimiento de la madera, y la industrialización.

Por su parte, la empresa forestal comunitaria de Agua Bendita solo cuenta con 71 ejidatarios y una superficie forestal de 2.218 ha (figura 1). Este ejido lidera la Unión de Ejidos Forestales Emiliano Zapata de Amanalco y se localiza fuera del polígono de la Reserva. Cuenta con bosques, aserraderos y comercializan la madera aserrada directamente a centros de comercialización a través de la Unión de Ejidos. El modelo de cadena productiva forestal de esta empresa es de producción (producción de planta, mantenimiento y protección), corte y abastecimiento, industrialización de la madera y comercialización bajo su control.

Instrumentos metodológicos de recolección de información. Se utilizó la observación directa y recorridos en campo, además, se aplicaron encuestas estructuradas, entrevistas semiestructuradas y entrevistas abiertas a diferentes actores como ejidatarios, mesa directiva, técnicos forestales y funcionarios públicos. Con estos instrumentos se obtuvo información cuantitativa (ingresos por la actividad forestal, número de hectáreas forestales aprovechables, edad promedio de ejidatarios, inversión, otras) y cualitativa (grado de organización, aspiraciones, conocimiento de objetivos de la empresa, liderazgo, otras) requerida.

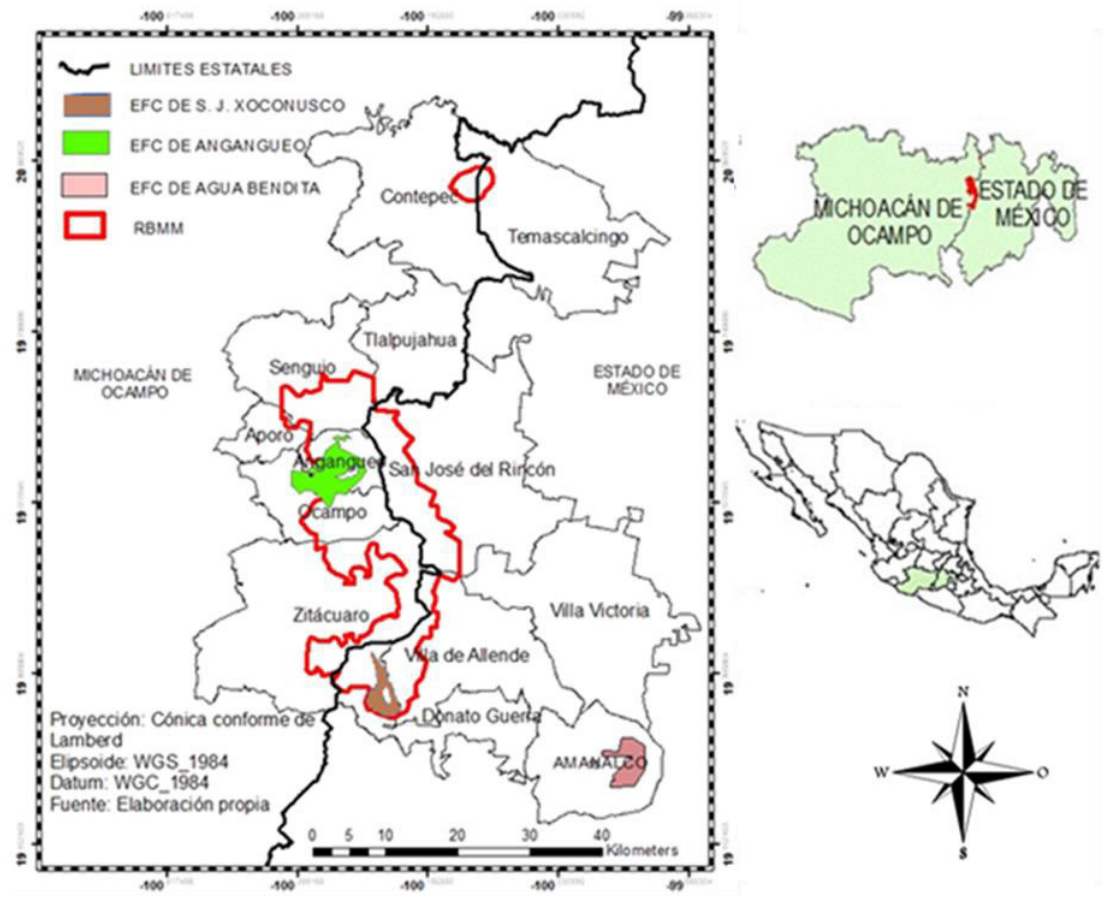

Figura 1. Localización geográfica de las tres empresas forestales estudiadas.

Geographical location of the three forestry enterprises studied. 
Determinación y aplicación de la muestra. Se calcularon los tamaños de muestra (n) para las tres poblaciones de ejidatarios con una fórmula de varianza máxima (Infante y Zárate 2012) (ecuación 1) de 60, 51 y 39 ejidatarios pertenecientes a las empresas forestales comunitarias de Angangueo $(\mathrm{N}=310)$, San Juan Xoconusco $(\mathrm{N}=211)$ y Agua Bendita $(\mathrm{N}=71)$, respectivamente.

$$
n=\frac{N Z^{2} p q}{N d^{2}+Z^{2} p q}
$$

Donde: $\mathrm{n}=$ Tamaño de la muestra; $\mathrm{N}=$ Tamaño de la población; $\mathrm{p}=$ Proporción de la población con una característica binomial; $\mathrm{q}=1-\mathrm{p} ; \mathrm{Z}^{2} \alpha=$ Valor de tabla de distribución normal Z (90\%); $\mathrm{d}^{2}=$ Error absoluto máximo deseado (fijado como una fracción de p) (10\%).

Impacto de la empresa forestal comunitaria en el manejo $y$ conservación de los recursos. La valoración ambiental incluyó tres fases:

(a) Percepción de los integrantes de la comunidad forestal sobre la conservación del recurso. En el diseño de la encuesta, se incluyó un conjunto de preguntas referidas al estado de conservación de las zonas de corta realizada en los últimos 10 años; grado de afectación del recurso agua, suelo y vida silvestre por la forma en cómo se realiza la actividad forestal en las áreas de corta; y actividades ilícitas ambientales en los terrenos forestales.

(b) Análisis multicriterio. Se diagnosticó el estado que guardan los recursos naturales de cada comunidad forestal de acuerdo a la opinión y experiencia de funcionarios públicos, técnicos y académicos que tienen alguna relación con dichas empresas por lo que se consideró un grupo interdisciplinario de 10 personas. Los criterios evaluados fueron: El grado de conservación (escala de 1 a 10) e importancia de éstos para la comunidad (1 a 5) en la consecución de proyectos productivos. La información generada se evaluó bajo la metodología de análisis multicriterio ( $\mathrm{Si}$ mon 1979, Prabhu et al. 1996) (ecuaciones 2 y 3).

$$
Y j=\frac{\sum_{i=1}^{n} X i j}{n}
$$

Donde: $X i j=$ Valor del experto i sobre el indicador $j(0 \leq$ $\mathrm{X} \leq 10) ; Y j=$ Valoración promedio del indicador $j$.

$$
E C_{R}=\sum_{j=1}^{m} \alpha j Y j
$$

Donde: $\alpha j=$ Ponderación asignada al indicador $j(0 \leq \alpha \leq 1)$; $\sum_{\mathrm{j}=1}^{\mathrm{m}} \alpha \mathrm{j}=1 ; E C_{R}=$ Estado de conservación del recurso natural $r$, el cual toma valores de 1 a 10 siendo 10 el máximo índice que puede tener la empresa forestal en cuanto a conservación del recurso, y 1 el peor.

(c) Generación de mapas. El análisis de la dinámica de la cubierta vegetal ofrece elementos para proveer evidencias cuantitativas del desempeño de las acciones de manejo y para proyectar tendencias. Las coberturas de bosque se mantienen en las áreas donde se realiza un manejo forestal comunitario con buena organización social (Palacio-Prieto et al. 2000, Lambin et al. 2001, Velázquez et al. 2002, DuránMedina et al. 2007). Se realizaron mapas de conflicto de uso de la tierra para las comunidades forestales de Angangueo y de San Juan Xoconusco, a través de la sobre posición de la capa de uso de suelo 2013 (clasificada en forestal, agrícola, pecuaria y otras) con la capa de uso potencial de la Reserva establecidos en el programa de manejo en el año 2000. La intersección dio como resultado un mapa del uso correcto o incorrecto de la tierra de la poligonal de la Reserva (DuranMedina et al. 2007). Se elaboró un mapa de detección de cambios de uso de la tierra de la Empresa Forestal Comunitaria de Agua Bendita, para ello se sobrepusieron dos mapas de distintos años (1987 y 2013). Se utilizó el conjunto vectorial de la carta de uso de la tierra y vegetación serie I (1987) y serie V (2013) y se reclasificó para cada uno de ellos en usos forestal, agrícola, pecuario y otros (figura 2). La elaboración de mapas y análisis de datos se realizó con el programa ArcGis® 9-ArcMap TM Versión 9.2.

Impacto socioeconómico de la integración de la cadena productiva forestal en los miembros de la empresa forestal comunitaria. La valoración de la parte socioeconómica comprendió tres fases:

(a) Elaboración de un índice de desarrollo local. Se construyó a partir de una base de datos del censo de población y vivienda 2010 del Instituto Nacional de Estadística y Geografía (INEGI 2010). El desarrollo social se puede medir a través de los indicadores de desarrollo humano y grado de marginación, este último en México se clasifica en muy alta, alta, media y baja en un intervalo numérico que va de $-1,3$ a 0,4 . En promedio, las comunidades rurales se ubican en intervalos de alta a muy alta marginación (INEGI 2010, CONAPO 2012). La selección de indicadores se ajustó a lo establecido por el Programa de las Naciones Unidas para el Desarrollo (PNUD 2014) y de la información disponible por el Instituto Nacional de Estadística y Geografía. Se eligieron 23 indicadores que fueron agrupados en las categorías de población, salud, equidad, educación y vivienda. (b) Aplicación de una encuesta. Se diseñó, construyó, calibró y aplicó una encuesta estructurada que incluyó un conjunto de preguntas dirigidas a los miembros de la empresa a efecto de obtener la información y opiniones de los socios sobre: conocimientos de los objetivos de la empresa, liderazgo, ingresos, mejoramiento de la condición de vida y otros temas.

(c) Análisis socioeconómico versus integración de la cadena productiva. La técnica multivariada de componentes principales se utilizó para elaborar un índice. Los componentes principales tienen como objetivos explorar las relaciones hipotéticas entre variables (Cortés y Rubalcava 1993). El componente principal $1\left(\mathrm{CP}_{1}\right)$ explica o resume la estructura subyacente de varianza y covarianza del con- 

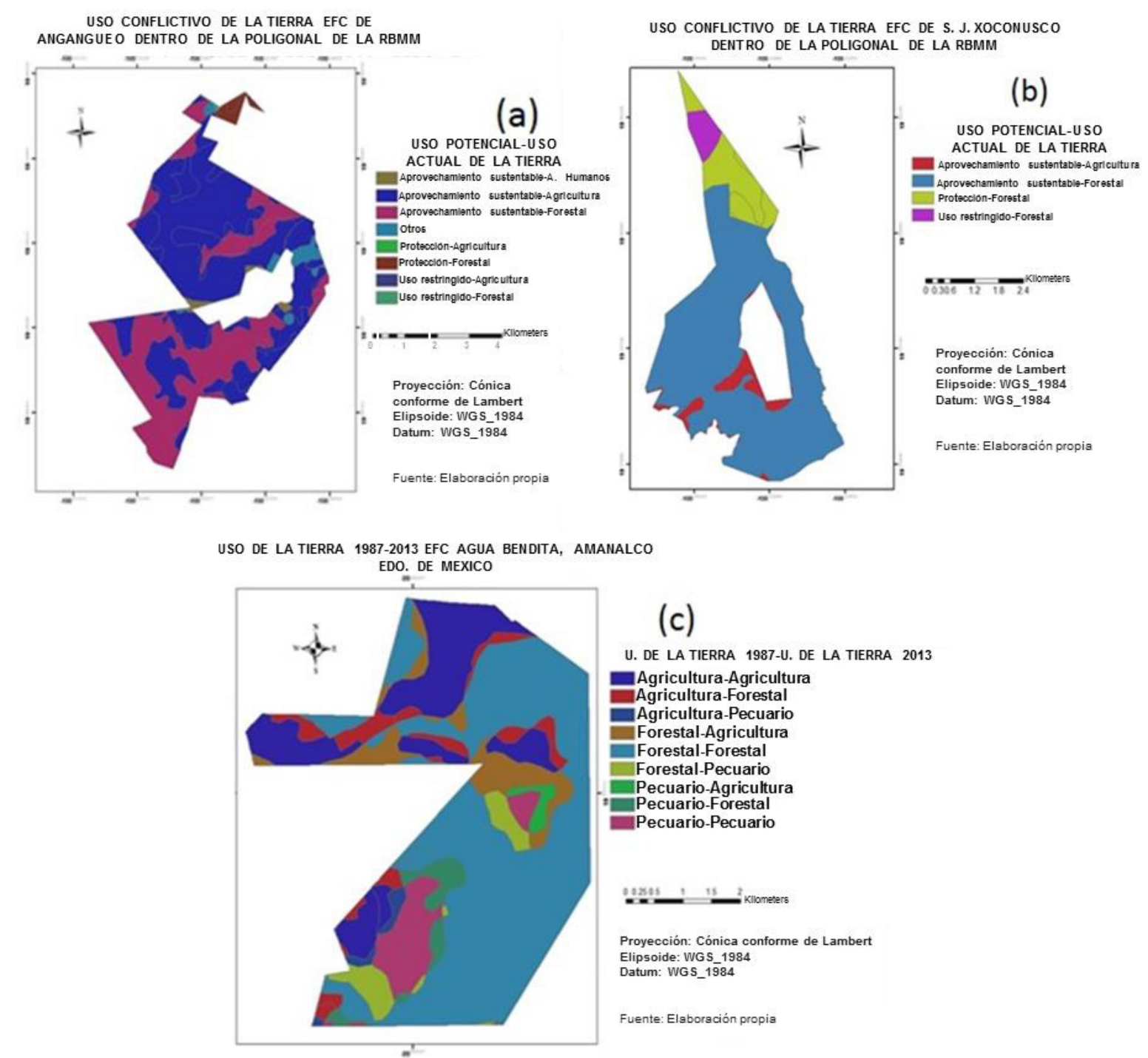

Figura 2. Mapas de uso conflictivo de la tierra de las empresas forestales de Angangueo (A) y San Juan Xoconusco (B), y del comportamiento del uso de tierra de Agua Bendita (1987-2013) (C).

Land use maps in conflict of the forest enterprises of Angangueo (A) and San Juan Xoconusco (B), and of the land use behavior of Agua Bendita (1987-2013) (C).

junto de indicadores (variables) a través de combinaciones lineales de estos por lo que sus valores sirven como parámetros comparativos de mayor, igual y menor. El algoritmo que calcula el índice de desarrollo local (IDL) es representado por el $\mathrm{CP}_{1}$ para cada comunidad o municipio (Blundell et al. 1998). En ese sentido, los IDL obtenidos para cada estudio de caso fueron: Angangueo 540, San Juan Xoconusco 646, y Agua Bendita 640 (ecuación 4).

$$
I D L=b_{1} X_{1}+b_{2} X_{2}+b_{3} X_{3}+\cdots+b_{n} X_{n}
$$

Donde: IDL $=$ Índice de desarrollo social; $X_{1}, X_{2}, X_{3}, \ldots, X_{n}$ $=$ Variables de desarrollo; $b_{1}, b_{2}, b_{3}, \ldots, b_{n}=$ multiplicadores o valores calculados para cada variable.

\section{RESULTADOS}

Visión empresarial. El cuadro 1 muestra que en la región de la Reserva existe una correspondencia entre el conocimiento de los principios corporativos de la comunidad forestal y niveles de integración de la cadena productiva. Cabe recordar que el ejido de Angangueo no ha logrado integrar una cadena productiva porque solo tiene la fase primaria del corte hasta el abasto, mientras que el ejido de San Juan Xoconusco ha integrado la industrialización básica con madera aserrada. Sin embargo, el ejido de Agua Bendita ha integrado una cadena productiva muy completa desde la fase primaria, industrialización y comercialización de productos primarios e industrializados y preside 
la Unión de Ejidos Forestales Emiliano Zapata ¿Por qué, entonces, se ha dado esa disparidad en la participación y desarrollo de las EFC a lo largo de la cadena productiva forestal?

Los resultados de la encuesta muestran diferencias significativas entre las tres empresas forestales comunitarias en términos de su capital social y principios corporativos. Los miembros de los ejidos de Angangueo (11\%), San Juan Xoconusco (73\%) y Agua Bendita (77 \%) señalaron que sí conocían los objetivos de la empresa forestal comunitaria. En ese mismo orden, los miembros de las empresas tienen la expectativa $(10 \%, 41 \%$ y $49 \%)$ de que la empresa estará mejor en los diez años próximos. La participación e involucramiento de los miembros de la organización en el proceso de toma de decisiones es un factor fundamental en la creación de una visión empresarial común que les permita trabajar juntos. Sin embargo, $38 \%$ de los miembros de la empresa forestal comunitaria de Angangueo opinó que nunca participa en la discusión de aspectos relacionados con los recursos forestales, una cantidad alta comparada con el $24 \%$ de San Juan Xoconusco y $8 \%$ de Agua Bendita.

Impacto de la empresa forestal comunitaria en el manejo y conservación de los recursos. (a) Percepción de los integrantes de la comunidad forestal respecto a la conservación del recurso. El cuadro 2 resume las frecuencias y porcentajes de la percepción de los miembros de las comunidades forestales referente a: (1) las condiciones de recuperación de las áreas de corta, y (2) el fomento a la conservación del recurso por la forma en cómo se aprovecha el recurso forestal maderable.

(1) Condiciones de recuperación de las áreas de corta. El $29 \%$ de los miembros de Angangueo piensa que sus áreas aprovechadas se encuentran en mal estado.

Cuadro 1. Principios corporativos que integran el capital social de las empresas forestales comunitarias.

Corporate principles that integrate social capital of community forestry enterprises.

\begin{tabular}{|c|c|c|c|c|c|c|c|}
\hline \multirow[t]{2}{*}{ Preguntas } & \multirow[t]{2}{*}{ Percepción } & \multicolumn{2}{|c|}{$\begin{array}{l}\text { Angangueo } \\
\quad(n=60)\end{array}$} & \multicolumn{2}{|c|}{$\begin{array}{l}\text { San Juan Xoconusco } \\
\qquad(\mathrm{n}=51)\end{array}$} & \multicolumn{2}{|c|}{$\begin{array}{l}\text { Agua Bendita } \\
\quad(\mathrm{n}=39)\end{array}$} \\
\hline & & $\mathrm{F}$ & $\% *$ & $\mathrm{~F}$ & $\% *$ & $\mathrm{~F}$ & $\% *$ \\
\hline \multirow{2}{*}{$\begin{array}{l}\text { Conocimiento de los objetivos de } \\
\text { la empresa forestal comunitaria }\end{array}$} & Sí los conocen & 7 & 11 & 37 & 73 & 30 & 77 \\
\hline & Desconocen & 53 & 89 & 14 & 27 & 9 & 23 \\
\hline \multirow{4}{*}{$\begin{array}{l}\text { Cómo se visualiza la organización } \\
\text { hacia el futuro ( } 10 \text { años) }\end{array}$} & Peor & 36 & 60 & 16 & 31 & 3 & 8 \\
\hline & Igual & 17 & 28 & 13 & 25 & 9 & 23 \\
\hline & Mejor & 6 & 10 & 21 & 41 & 19 & 49 \\
\hline & Mucho mejor & 1 & 2 & 1 & 2 & 8 & 21 \\
\hline \multirow{4}{*}{$\begin{array}{l}\text { Involucramiento respecto asuntos } \\
\text { forestales en las reuniones ejidales }\end{array}$} & Siempre participa & 19 & 32 & 22 & 43 & 23 & 59 \\
\hline & Rara vez participa & 14 & 23 & 15 & 29 & 12 & 31 \\
\hline & Nunca participa & 23 & 38 & 12 & 24 & 3 & 8 \\
\hline & Son asuntos de la autoridad ejidal & 4 & 7 & 2 & 4 & 1 & 3 \\
\hline \multirow{3}{*}{ Cómo estaba el ejido hace 10 años } & Mejor que ahora & 38 & 63 & 25 & 49 & 8 & 21 \\
\hline & Igual que ahora & 17 & 28 & 12 & 24 & 15 & 38 \\
\hline & Peor que ahora & 5 & 9 & 14 & 27 & 16 & 41 \\
\hline \multirow{3}{*}{$\begin{array}{l}\text { Cuál es la opción que prefiere para } \\
\text { el recurso forestal }\end{array}$} & Aprovechar & 20 & 33 & 19 & 37 & 8 & 21 \\
\hline & Conservar & 35 & 58 & 23 & 45 & 1 & 3 \\
\hline & Aprovechar y conservar & 5 & 8 & 9 & 18 & 30 & 77 \\
\hline \multirow{3}{*}{ Convivencia de la comunidad } & Alta & 21 & 35 & 7 & 14 & 12 & 31 \\
\hline & Media & 34 & 57 & 39 & 76 & 23 & 59 \\
\hline & Baja & 5 & 8 & 5 & 10 & 4 & 10 \\
\hline \multirow{3}{*}{ Liderazgo de sus dirigentes } & Buena & 9 & 15 & 10 & 20 & 19 & 49 \\
\hline & Regular & 39 & 65 & 37 & 73 & 17 & 44 \\
\hline & Mala & 12 & 20 & 4 & 8 & 3 & 8 \\
\hline
\end{tabular}

*Valores redondeados; $\mathrm{F}=$ frecuencias y $\mathrm{n}=$ número de encuestados. 
En contraste, el $100 \%$ de los integrantes de San Juan Xoconusco opina que las suyas se encuentran entre regulares a buenas; mientras que el $95 \%$ de los de Agua Bendita considera que sus áreas bajo aprovechamiento van de regulares $(21 \%)$, buenas $(64 \%)$ y excelentes (10\%). En general, el $64 \%$ de los miembros de Agua Bendita tiene la apreciación más alta de que las áreas de corta recientes se encuentran en buenas condiciones de recuperación, en comparación con el $34 \%$ y $43 \%$ de Angangueo y San Juan Xoconusco, respectivamente (cuadro 2 y figura 3 ).

Otro indicador de la responsabilidad social de la comunidad forestal en la conservación del bosque lo constituye la participación de los miembros de la comunidad forestal en acciones destinadas a la recuperación y conservación en las áreas aprovechadas. En ese sentido, las comunidades forestales de San Juan Xoconusco y Agua Bendita son muy parecidas, ambas realizan casi

Cuadro 2. Conservación de los recursos naturales por la forma de aprovechar el recurso forestal.

Natural resources conservation by the way to use them.

\begin{tabular}{|c|c|c|c|c|c|c|c|c|c|c|}
\hline \multirow{3}{*}{ Empresas forestales comunitarias } & \multirow{2}{*}{\multicolumn{3}{|c|}{$\begin{array}{l}\text { Condiciones de recuperación } \\
\text { de las áreas de corta }\end{array}$}} & \multicolumn{7}{|c|}{$\begin{array}{l}\text { Fomento a la conservación del recurso por la forma en cómo se } \\
\text { aprovecha el recurso forestal maderable }\end{array}$} \\
\hline & & & & \multirow{2}{*}{ Respuesta } & \multicolumn{2}{|c|}{ Agua } & \multicolumn{2}{|c|}{ Suelo } & \multicolumn{2}{|c|}{ Vida silvestre } \\
\hline & Respuesta & $\mathrm{F}$ & $\%$ & & $\mathrm{~F}$ & $\%$ & $\mathrm{~F}$ & $\%$ & $\mathrm{~F}$ & $\%$ \\
\hline \multirow{3}{*}{ Angangueo } & Malas & 17 & 29 & Sí & 15 & 26 & 18 & 32 & 17 & 31 \\
\hline & Regulares & 21 & 36 & Poco & 3 & 5 & 3 & 5 & 4 & 7 \\
\hline & Buenas & 20 & 34 & No & 38 & 67 & 36 & 63 & 34 & 62 \\
\hline \multirow{3}{*}{ San Juan Xoconusco } & Malas & 0 & 0 & Sí & 19 & 37 & 25 & 49 & 17 & 33 \\
\hline & Regulares & 29 & 57 & Poco & 4 & 8 & 5 & 10 & 6 & 12 \\
\hline & Buenas & 22 & 43 & No & 28 & 55 & 21 & 41 & 28 & 55 \\
\hline \multirow{4}{*}{ Agua Bendita } & Malas & 2 & 5 & Sí & 37 & 95 & 35 & 90 & 29 & 74 \\
\hline & Regulares & 8 & 21 & Poco & 0 & 0 & 2 & 5 & 6 & 15 \\
\hline & Buenas & 25 & 64 & No & 2 & 5 & 2 & 5 & 4 & 10 \\
\hline & Excelente & 4 & 10 & - & - & - & - & - & - & - \\
\hline
\end{tabular}

$\mathrm{F}=$ frecuencias

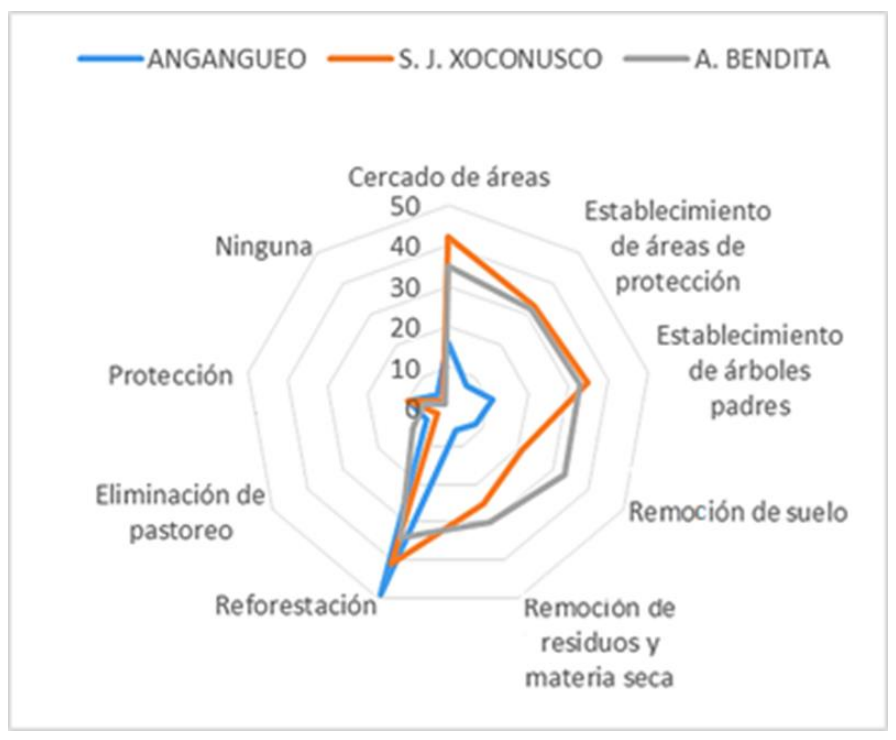

Figura 3. Actividades que la empresa forestal realiza para recuperar las áreas intervenidas.

Activities that the forestry enterprise performs to recover affected areas. 
el doble de acciones que Angangueo y diversifican sus acciones en diferentes tareas de recuperación de las áreas perturbadas (figura 3 ).

(2) Fomento a la conservación del recurso por la forma en cómo se aprovecha el recurso forestal maderable. Los integrantes de las empresas poseen una apreciación de fomento diferente para cada uno de los recursos: agua, suelo y vida silvestre por la actividad forestal. Por ejemplo, para Angangueo: $26 \%, 32 \%$ y $31 \%$; San Juan Xoconusco: 37 \%, 49 \% y $33 \%$; y para Agua Bendita: $95 \%$, $90 \%$ y $74 \%$, respectivamente. Bajo este indicador, la comunidad forestal de Agua Bendita es la mejor posicionada, seguida de San Juan Xoconusco y Angangueo (cuadro 2).

(b) Opinión de expertos sobre la conservación de los recursos forestales de las comunidades. Los recursos con mayor importancia fueron: El suelo, el agua y el bosque. En contraste, el de menor importancia fue el conocimiento ancestral. En cuanto a la calificación del grado de conservación o variación del recurso: la comunidad forestal de Agua Bendita fue la que mejor ha conservado su bosque y no parece haber sufrido cambios significativos en la variación de su superficie forestal (cuadro 3). En ese sentido, de acuerdo al índice de conservación generado, la comunidad forestal de Agua Bendita fue la más favorecida $(8,27)$, seguida por San Juan Xoconusco $(8,18)$ y Angangueo $(7,44)$ (c) Análisis espacial. El cuadro 4 muestra los resultados de la superficie de uso agrícola, forestal y de asentamientos humanos que actualmente tiene cada comunidad. Los usos agrícolas y de asentamientos humanos son prohibidos en terrenos bajo aprovechamiento forestal sustentable. La empresa de Angangueo en estos terrenos perdió 1.756,11 ha (disminución de $63,19 \%$ ), constituidas por: agricultura $1.729,20$ ha y asentamientos humanos 26,91 ha. Por su parte, la empresa de San Juan Xoconusco solo perdió 84,04 ha (disminución de 6,16\%), las cuales utilizó para agricultura.

El cuadro 5 resume matricialmente las diferencias entre el uso de la tierra en 2013 en comparación al tenido en 1987 para la empresa forestal comunitaria de Agua Bendita. Se muestran las superficies estables (valores en la diagonal) y dinámicas (valores no diagonales), así como las probabilidades de mantenerse (diagonales) o de cambio de usos (no diagonales); por ejemplo, la superficie forestal que no cambió de uso de 1987 a 2013 es de 1.182,56 ha, y la probabilidad de que una hectárea de uso forestal se mantenga en el mismo uso es de $86,8 \%$. Durante ese periodo, la superficie forestal obtuvo una ganancia de 305,19 ha provenientes de terrenos agrícolas $(211,10 \mathrm{ha})$ y pecuarios $(94,09 \mathrm{ha})$. Sin embargo, la superficie forestal también sufrió una pérdida de 179,58 ha, de las cuales 112,90 y 66,68 ha pasaron a tener usos agrícolas y pecuarios, respectivamente.

Impacto socioeconómico de la integración de las cadenas productivas en las empresas forestales comunitarias. Más de $50 \%$ de los miembros de las empresas estudiadas per-

Cuadro 3. Análisis multicriterio sobre el estado de conservación del recurso forestal en las tres empresas forestales comunitarias.

A multi-criteria analysis on the conservation state of forest resources in the three forest community enterprises.

\begin{tabular}{|c|c|c|c|c|c|c|}
\hline \multirow[t]{2}{*}{ Recurso natural (ponderación = $\alpha$ ) } & \multicolumn{3}{|c|}{$Y_{j}=\frac{\sum_{i=1}^{n} x_{i j}}{n}$} & \multicolumn{3}{|c|}{$\sum_{j=1}^{m} \alpha_{j} Y_{j}$} \\
\hline & ANG & SJX & AGB & ANG & SJX & AGB \\
\hline Suelo $(0,161290323)$ & 6,60 & 8,00 & 8,50 & 1,0645 & 1,29032 & 1,3710 \\
\hline Agua $(0,161290323)$ & 7,40 & 8,83 & 8,50 & 1,1935 & 1,42473 & 1,3710 \\
\hline Flora y fauna $(0,096774194)$ & 7,40 & 8,50 & 8,00 & 0,7161 & 0,82258 & 0,7742 \\
\hline Bosque $(0,129032258)$ & 7,80 & 8,67 & 9,17 & 1,0065 & 1,11828 & 1,1828 \\
\hline Belleza escénica $(0,096774194)$ & 8,20 & 7,50 & 7,83 & 0,7935 & 0,72581 & 0,7581 \\
\hline Variación de la superficie forestal $(0,096774194)$ & 6,60 & 8,00 & 8,67 & 0,6387 & 0,77419 & 0,8387 \\
\hline Conocimiento ancestral $(0,064516129)$ & 7,20 & 7,00 & 7,50 & 0,4645 & 0,45161 & 0,4839 \\
\hline Investigación (0,096774194) & 8,00 & 7,67 & 7,17 & 0,7742 & 0,74194 & 0,6935 \\
\hline Conflicto de uso de suelo $(0,096774194)$ & 8,20 & 8,67 & 8,33 & 0,7935 & 0,83871 & 0,8065 \\
\hline$\beta=\sum_{j=1}^{m} \alpha_{j} Y_{j}$ & & & & 7,4451 & 8,1881 & 8,2796 \\
\hline
\end{tabular}

$\mathrm{ANG}=$ Angangueo $; \mathrm{SJX}=$ San Juan Xoconusco $; \mathrm{AGB}=$ Agua Bendita $; j=$ Valor promedio para el recurso $j ; \alpha=$ ponderación asignada al recurso $j$; $\beta=$ índice de conservación. 
Cuadro 4. Resumen de uso actual versus uso potencial de la tierra de las comunidades forestales de Angangueo y San Juan Xoconusco.

Summary of current use versus potential land use in the land of the forest communities of Angangueo and San Juan Xoconusco.

\begin{tabular}{|c|c|c|c|c|c|c|c|c|}
\hline \multirow{3}{*}{ Uso potencial (año 2000) } & \multicolumn{8}{|c|}{ Uso de la tierra (año 2013) } \\
\hline & \multicolumn{2}{|c|}{ Agricultura } & \multicolumn{2}{|c|}{ Forestal } & \multicolumn{2}{|c|}{$\begin{array}{l}\text { Asentamientos } \\
\text { humanos }\end{array}$} & \multicolumn{2}{|c|}{$\begin{array}{c}\text { Total } \\
\text { (Uso potencial) }\end{array}$} \\
\hline & ANG & SJX & ANG & SJX & ANG & SJX & ANG & SJX \\
\hline Aprovechamiento sustentable & $1.729,200$ & 84,04 & 1.023 .00 & $1.280,10$ & 26,91 & & $2.779,10$ & $1.364,20$ \\
\hline Protección & 0,305 & & 45,74 & 184,82 & & & 46,04 & 184,82 \\
\hline Uso restringido & 0,026 & & 2,77 & 55,30 & & & 2,79 & 55,30 \\
\hline Otros & & & & & & & 68,64 & \\
\hline Total & $1.729,58$ & 84,04 & $1.071,51$ & $1.520,20$ & 26,91 & & $2.896,57$ & $1.604,30$ \\
\hline
\end{tabular}

ANG = Angangueo; SJX = San Juan Xoconusco; AGB = Agua Bendita .

Cuadro 5. Matriz de intercambio de uso de la tierra y probabilidades de transición de usos durante el periodo 1987-2013 en la comunidad forestal de Agua Bendita.

Matrix of exchange of land use and probabilities of transition of use during the period 1987-2013 in the forest community of Agua Bendita.

\begin{tabular}{|c|c|c|c|c|c|c|c|c|c|}
\hline \multirow[b]{2}{*}{ Uso de la tierra 1987} & \multicolumn{4}{|c|}{ Uso de la tierra 2013} & \multirow[b]{2}{*}{$\%$} & \multicolumn{4}{|c|}{ Probabilidad de cambios de uso de la tierra } \\
\hline & $\begin{array}{c}\text { Agrícola } \\
\text { (ha) }\end{array}$ & $\begin{array}{c}\text { Forestal } \\
\text { (ha) }\end{array}$ & $\begin{array}{c}\text { Pecuario } \\
\text { (ha) }\end{array}$ & $\begin{array}{l}\text { Total } \\
\text { (ha) }\end{array}$ & & $\begin{array}{c}\text { Agrícola } \\
(\%)\end{array}$ & $\begin{array}{c}\text { Forestal } \\
(\%)\end{array}$ & $\begin{array}{c}\text { Pecuario } \\
(\%)\end{array}$ & $\begin{array}{c}\text { Total } \\
(\%)\end{array}$ \\
\hline Agrícola & 371,04 & 211,10 & 23,07 & 605,21 & 27,3 & 61,3 & 34,9 & 3,8 & 100,0 \\
\hline Forestal & 112,90 & $1.182,56$ & 66,68 & $1.362,14$ & 61,4 & 8,3 & 86,8 & 4,9 & 100,0 \\
\hline Pecuario & 13,48 & 94,09 & 143,61 & 251,17 & 11,3 & 5,4 & 37,5 & 57,2 & 100,0 \\
\hline Total & 497,42 & $1.487,75$ & 233,36 & $2.218,53$ & 100,0 & 22,4 & 67,1 & 10,5 & 100,0 \\
\hline
\end{tabular}

ciben que dicha actividad representa menos del $25 \%$ de sus ingresos. Sin embargo, un análisis más detallado de los datos sugiere que la actividad forestal representa una ayuda significativa para los miembros en la medida que se integran verticalmente las empresas forestales comunitarias ya que existe una relación entre ingreso mensual general e ingreso anual por la actividad forestal (relación logarítmica $\left.\mathrm{r}^{2}=0,67\right)$. La aseveración se fortalece porque el $27 \%$, $35 \%$ y $46 \%$ de los encuestados de Angangueo, San Juan Xoconusco y Agua Bendita respectivamente, perciben que la actividad forestal contribuye por encima del $25 \%$ a sus ingresos. La aportación se incrementa en la medida que se integran más eslabones al modelo de la cadena productiva (cuadro 6). Las empresas de Angangueo, San Juan Xoconusco y Agua Bendita aportan 7,4 \%, 9,5 \% y $18,38 \%$ de los ingresos de la actividad forestal a obra social (cuadro 6).

La figura 4 muestra en una gráfica de radar los porcentajes de inversión destinados a cada tipo de obra. Agua Bendita es la comunidad que más diversifica el apoyo a obras sociales, por ejemplo envía el $99 \%$ de los recursos a los rubros: clínicas (4 \%), caminos (18\%), salón de usos

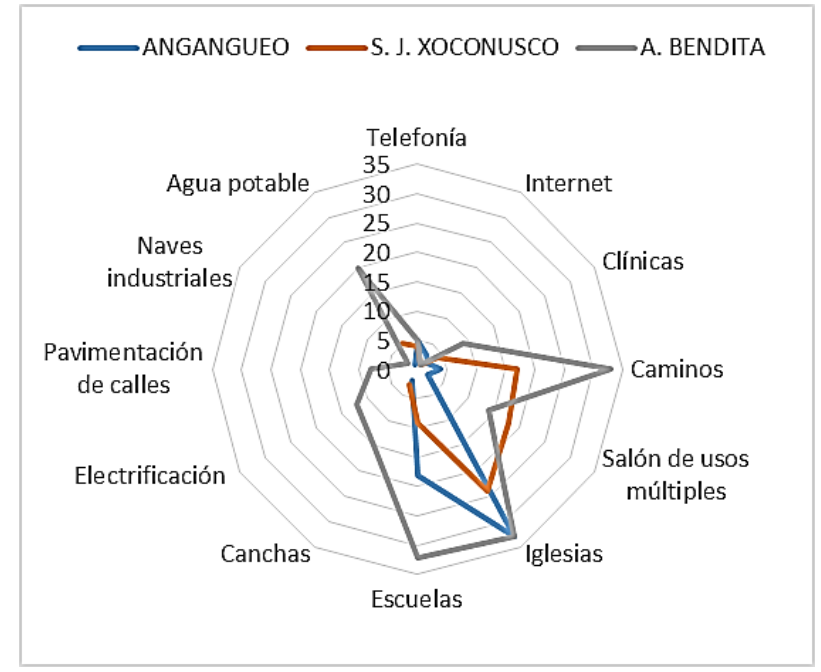

Figura 4. Destinos de los recursos procedentes de la empresa forestal hacia obra social.

Destination of resources coming from the forestry enterprise towards social work. 
múltiples (8\%), iglesias (19\%), escuelas (18\%), canchas $(8 \%)$, electrificación (7\%), pavimentación de calles (4 $\%)$, naves industriales (1\%) y agua potable (12\%). En estos mismos rubros para San Juan Xoconusco y Angangueo fueron: San Juan Xoconusco $89 \%$ en: clínicas (3\%), caminos $(20 \%)$, salón de usos múltiples (23\%), iglesias $(30 \%)$, escuelas $(12 \%)$, canchas $(1 \%)$ y los demás $(0$ $\%$ ); y Angangueo $89 \%$ en: clínicas (3\%), caminos (6\%), salón de usos múltiples (3\%), iglesias (47\%), escuelas (26 $\%)$, canchas (3\%), agua potable (1\%) y los demás (0\%). En los tres estudios de caso, la iglesia es la que más apoyo recibe de la actividad forestal porque es la institución más fuerte dentro de las comunidades ya que congrega las celebraciones tradicionales y fiestas religiosas, por lo que juega un papel importante en la convivencia y cohesión social de la comunidad rural.

Se calculó el índice de marginación de cada una de las tres comunidades en tres tiempos. Cabe señalar que este índice tiene una connotación negativa, entre mayor sea el valor numérico, peor situación económica tiene una comunidad. En la figura 5 se muestran los valores calculados para los años 1990, 2000 y 2010. Las tres empresas forestales comunitarias estudiadas tuvieron una tendencia similar a empeorar su situación de bienestar entre los años

Cuadro 6. Indicadores económicos de las comunidades forestales de estudio.

Summary table of economic indicators of forest communities studied.

\begin{tabular}{|c|c|c|c|c|c|c|}
\hline Indicador & \multicolumn{2}{|c|}{$\begin{array}{l}\text { Angangueo } \\
(\mathrm{n}=60)\end{array}$} & \multicolumn{2}{|c|}{$\begin{array}{l}\text { San Juan Xoconusco } \\
\qquad(\mathrm{n}=51)\end{array}$} & \multicolumn{2}{|c|}{$\begin{array}{l}\text { Agua Bendita } \\
\quad(n=39)\end{array}$} \\
\hline Ingresos anual por actividad forestal & \multicolumn{2}{|c|}{$\begin{array}{l}x=4.500,00 \\
\mathrm{ds}=1.498,2\end{array}$} & \multicolumn{2}{|c|}{$\begin{aligned} \mathrm{x} & =5.240,00 \\
\mathrm{ds} & =3.7022,00\end{aligned}$} & \multicolumn{2}{|c|}{$\begin{aligned} \mathrm{x} & =8.420,00 \\
\mathrm{ds} & =4.3155,00\end{aligned}$} \\
\hline Ingresos mensual general & \multicolumn{2}{|c|}{$\begin{array}{l}x=2.439,10 \\
(\mathrm{ds}=222,7)\end{array}$} & \multicolumn{2}{|c|}{$\begin{array}{c}x=3.670,81 \\
(\mathrm{ds}=3.514,6)\end{array}$} & \multicolumn{2}{|c|}{$\begin{array}{c}x=4.000,82 \\
(\mathrm{ds}=3.885,6)\end{array}$} \\
\hline \multirow{2}{*}{$\begin{array}{l}\text { Percepción de mejoramiento de vida por la } \\
\text { actividad forestal }\end{array}$} & $\mathrm{Si}$ & $54 \%$ & $\mathrm{Si}$ & $70 \%$ & $\mathrm{Si}$ & $90 \%$ \\
\hline & No & $46 \%$ & No & $30 \%$ & No & $10 \%$ \\
\hline \multirow{4}{*}{$\begin{array}{l}\text { Percepción sobre aportación a la economía } \\
\text { familiar }\end{array}$} & $<25 \%$ & $73 \%$ & $<25 \%$ & $65 \%$ & $<25 \%$ & $54 \%$ \\
\hline & $25 \%-50 \%$ & $17 \%$ & $25 \%-50 \%$ & $27 \%$ & $25 \%-50 \%$ & $17 \%$ \\
\hline & $>50 \%$ & $6 \%$ & $>50 \%$ & $8 \%$ & $>50 \%$ & $29 \%$ \\
\hline & Nada & $4 \%$ & Nada & $0 \%$ & Nada & $0 \%$ \\
\hline $\begin{array}{l}\text { Porciento de ingresos de la empresa forestal } \\
\text { comunitaria que se destina a obra social }\end{array}$ & \multicolumn{2}{|c|}{$\begin{array}{c}\mathrm{n}=11 \\
\mathrm{x}=7,4 \\
\mathrm{ds}=4,8\end{array}$} & \multicolumn{2}{|c|}{$\begin{array}{c}\mathrm{n}=28 \\
\mathrm{x}=9,5 \\
\mathrm{ds}=7,9\end{array}$} & \multicolumn{2}{|c|}{$\begin{array}{c}\mathrm{n}=32 \\
\mathrm{x}=18,51 \\
\mathrm{ds}=7,23\end{array}$} \\
\hline
\end{tabular}

$\mathrm{n}=$ número de la muestra; $\mathrm{x}=$ media; ds = desviación estándar.

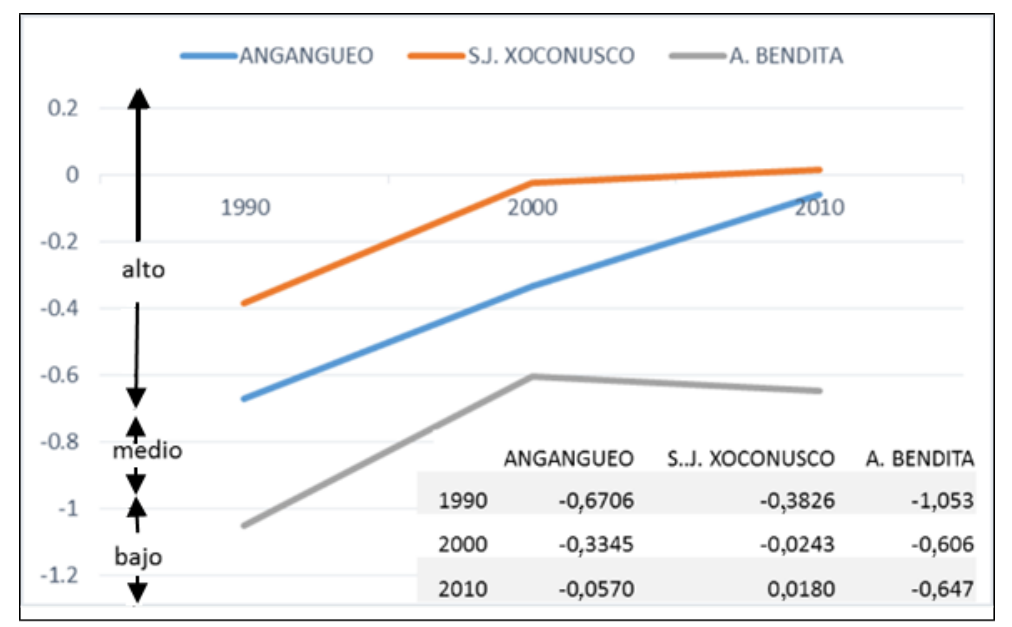

Figura 5. Comportamiento del índice de marginación de las tres empresas estudiadas.

Marginalization index of the three enterprises studied. Source: INEGI (2010). 
1990 y 2000. Entre el 2000 y el 2010, solo Agua Bendita disminuyó su marginación social (de -0,606 a -0,647), esto es, que sus condiciones de bienestar mejoraron. San Juan Xoconusco tuvo una ligera caída en su bienestar entre esos mismos años (de - 0,0243 a 0,0180 ). En cambio, Angangueo siguió la misma tendencia negativa mostrada en la década anterior (de $-0,3345$ a -0,0570) empeorando las condiciones sociales de sus integrantes.

\section{DISCUSIÓN}

Las tres empresas forestales comunitarias estudiadas tienen diferentes niveles de integración en sus cadenas productivas en la región de la Reserva de la Biosfera de la Mariposa Monarca. El estudio permitió identificar las diferencias en la cultura corporativa de sus integrantes que forman su capital social. La empresa forestal comunitaria de Agua Bendita fue la que mayormente integra su cadena y sus integrantes son quienes conocen más los objetivos y los principios empresariales corporativos de la misma. Contrariamente, la empresa de Angangueo se encuentra en ese proceso y sus miembros se conducen con menos principios corporativos empresariales. De aquí, se desprende la interrogante siguiente: ¿Qué fue primero: la integración de la cadena productiva o la cultura corporativa de la empresa forestal comunitaria que desarrollaron sus integrantes? Los datos de campo (conocimiento de objetivos, optimismo, liderazgo, participación y convivencia comunitaria (cuadro 1)) sugieren que la integración de los principios de la cultura corporativa se formó antes que la integración de la cadena productiva y, posteriormente, estos principios continuaron consolidándose con el funcionamiento de los eslabones integrados. Esto concuerda con los hallazgos de Garibay (2005) que señala que no todos los ejidos o comunidades agrarias que tienen bosques han podido desarrollar una empresa forestal comunitaria exitosa a pesar que muchos ejidos recibieron apoyos gubernamentales para adquirir maquinaria para industrializar o para comercializar sus productos porque no pudieron consolidar su capital social bajo los principios corporativos empresariales.

Bray y Merino (2005) mencionan que, en México, la mayor parte de las comunidades forestales se asemejan al ejido de Angangueo donde solo se dedican a la producción forestal y una minoría participa y controla el abastecimiento de madera, asimismo cuestionan que a ejidos como el de Angangueo se les llame empresas comunitarias forestales porque no tienen una verdadera actividad productiva y no llevan un negocio formal. Si se aplica este criterio, estaría en duda denominar a la comunidad de Angangueo como empresa forestal comunitaria, en cambio, se le debería llamar comunidad forestal rentista porque solo cobra una renta a alguien que pueda cortar los árboles de sus tierras. La comunidad de Angangueo muestra una carencia de una visión empresarial, carencia de un liderazgo visionario, la falta de interés y los conflictos internos que no permiten a los miembros trabajar juntos.
En la empresa forestal comunitaria de San Juan Xoconusco se encontró la presencia de principios corporativos empresariales que les permitieron avanzar de la producción primaria y el abasto forestal hacia el eslabón de la transformación; sin embargo, las relaciones comerciales son controladas por agentes económicos externos que les imponen las condiciones de comercialización. Finalmente, la empresa comunitaria forestal de Agua Bendita presentó los niveles más altos de conocimiento y aplicación de los principios corporativos. Los ejidatarios reconocen que el liderazgo visionario tuvo un gran rol en la creación de principios corporativos que permitieron al ejido de Agua Bendita integrar todos los eslabones de la cadena y ser una comunidad líder en la región al presidir la organización de segundo nivel (Unión de Ejidos Forestales Emiliano Zapata) que tiene como principal objetivo comercializar los productos maderables y gestionar apoyos gubernamentales.

Los principios corporativos y la mayor integración de la empresa forestal comunitaria de Agua Bendita se ven reflejados en una mejor calidad de las actividades productivas y la conservación de sus recursos naturales especialmente en las áreas que han sido perturbadas con el corte de madera. En cambio, la comunidad forestal de Angangueo puso menor esfuerzo en conservar sus recursos en las áreas aprovechadas para madera y realizó menos actividades de conservación en el resto de sus bosques. Aquí está claro que la conservación del capital natural, mediante la realización de buenas prácticas de manejo forestal, tiene una relación directa con una mejor organización y una integración vertical de las organizaciones sociales forestales.

El estudio de la dinámica de la cubierta vegetal es un signo evidente de la realización de buenas prácticas de manejo forestal y de tendencias. Los resultados de la investigación muestran que la comunidad de Angangueo tuvo una disminución muy significativa de la superficie del uso de la tierra para aprovechamiento forestal sustentable, $(63,2 \%)$ del año 2000 al 2013, a pesar que está dentro del polígono de la Reserva de la Biosfera de la Mariposa Monarca que marca restricciones importantes para realizar actividades productivas, tanto en la zona de amortiguamiento como en la zona núcleo de esta área natural protegida. Por el contrario, la comunidad forestal de Agua Bendita amplió su superficie forestal $(4,9 \%)$ recuperándola de zonas destinadas a la agricultura y a la ganadería; además, esta empresa tiene una probabilidad muy alta $(86,8 \%)$ de mantener sus terrenos con el uso forestal actual y una probabilidad baja $(8,3 \%)$ de que estos cambien a un uso agrícola. Esto es importante si se considera que esta comunidad está fuera del área natural protegida y no está obligada a mantener o incrementar su superficie forestal. Las diferencias se hicieron más evidentes cuando se realizó el cálculo propuesto del índice multicriterio de conservación de los recursos forestales (cuadro 3) en el cual Agua Bendita tuvo el mayor índice, seguido por San Juan Xoconusco y, al final, Angangueo con el índice menor, indicando que a mayor índice, mayor grado de conservación. 
Una explicación a estas diferencias se encuentra en el éxito económico logrado por una empresa forestal comunitaria como la de Agua Bendita, que con la producción y venta de sus productos maderables hace que sus integrantes tengan un mayor interés en invertir en recursos bienes de capital y capital humano (factores de producción avanzados) y en conservar su capital natural (factores de producción básicos).

El éxito económico también debe reflejarse tanto en el mejoramiento del ingreso de los socios por la actividad forestal como en las mejoras en la comunidad que al final se traduce en un mejor nivel de vida. Angangueo tuvo el menor ingreso por socio, el menor porcentaje de inversión en obras sociales en la localidad y la percepción más baja en el nivel de vida; San Juan Xoconusco obtuvo los tres indicadores más elevados, mientras que Agua Bendita fue la empresa forestal comunitaria con mayores indicadores de mejoramiento económico. El cálculo de un indicador contrapuesto al anterior permitió corroborar las diferencias entre las tres comunidades, se calculó el nivel de marginación social entre 1990 y 2010 y se observó que Angangueo empeoró significativamente, San Juan Xoconusco también empeoró ligeramente; por el contario, Agua Bendita disminuyó su índice de marginación social.

Es importante señalar que los recursos económicos generados por la comunidad forestal en la región de la Reserva son significativos para la economía de los miembros. En el estudio realizado se calcularon los ingresos per capita de las tres empresas, obteniendo ingresos por $\$ 4.500$ (US\$ 243,2 ), \$ 5.240 (US\$283,1) y $\$ 8.420$ (US\$455,0) pesos anuales en promedio por la actividad forestal en las comunidades forestales de Angangueo, San Juan Xoconusco y Agua Bendita, respectivamente. En las tres comunidades tuvieron ingresos mayores al ingreso mensual per cápita para una comunidad rural en México que en 2016 fluctúo entre \$2.700 (US\$ 145,8) y $\$ 6.799$ (US\$ 367,0) (CONASAMI 2016). Se observa nuevamente la misma tendencia entre las comunidades forestales estudiadas, los socios de la empresa forestal comunitaria de Agua Bendita, que es la de mayor integración de su cadena productiva, fueron los que tuvieron el mayor ingreso, seguidos de los de San Juan Xoconusco y al final estuvo la de Angangueo.

Las empresas forestales comunitarias además de los beneficios monetarios, también proveen a sus socios otros bienes de consumo como la leña para combustible y productos comestibles (como hongos y herbáceas). Estos apoyan su economía, además de estar íntimamente ligados a sus prácticas culturales tradicionales y vida diaria.

Por su carácter comunitario, las empresas forestales comunitarias estudiadas son un ejemplo de institución que bien puede considerarse con responsabilidad social y ambiental, en la medida que participa en niveles superiores de la cadena productiva forestal. Por un lado, dicho compromiso lo materializa en la aportación de recursos económicos para obras sociales. Y por otro, como lo mencionan Rodríguez et al. (2018) la participación de los miembros de la comunidad en la realización de acciones destinadas para asegurar la recuperación y conservación en las áreas aprovechadas se incrementan en la medida que se integran eslabones a la CPF.

\section{CONCLUSIONES}

En las empresas forestales comunitarias estudiadas de la Reserva de la Biosfera de la Mariposa Monarca existe una correspondencia entre el capital social expresado en el grado de conocimiento de los principios corporativos o cultura empresarial y el grado de integración de la cadena productiva forestal. La empresa forestal comunitaria de Agua Bendita es la que posee mayor cultura empresarial siendo la que ha integrado más eslabones de la cadena productiva forestal; la comunidad de San Juan Xoconusco tiene una integración de la fase primaria y la industrialización y una cultura empresarial en desarrollo; mientras que la comunidad de Angangueo es la que presenta una menor cultura empresarial y la que tiene solo el eslabón de la fase primaria de producción de madera, por lo cual, en sentido estricto, no debería ser considerada empresa forestal comunitaria.

Se concluye que a mayor grado de integración de la cadena productiva, las empresas forestales comunitarias de la Reserva de la Biosfera de la Mariposa Monarca tienden a realizar más y mejores prácticas de conservación del recurso forestal que se expresan en incrementos de la superficie de cubierta forestal de las tierras de las empresas. La comunidad de Angangueo ha sufrido un deterioro importante de sus recursos forestales, en tanto que la empresa de San Juan Xoconusco ha tenido un deterioro mínimo. Por el contrario, la empresa forestal comunitaria de Agua Bendita incrementa su superficie arbolada.

En las empresas comunitarias forestales estudiadas también existe correspondencia directa entre el grado de integración de la cadena productiva y el beneficio económico individual de los socios y el beneficio social para las comunidades.

Por estos hallazgos, se recomienda que las políticas públicas en México fomenten los principios corporativos de las comunidades forestales del capital humano y capital social antes o al mismo tiempo que otorguen apoyos económicos que mejoren los bienes de capital o el capital natural que permitan la integración de una cadena productiva maderable y la formación de verdaderas empresas forestales comunitarias.

\section{REFERENCIAS}

Antinori C. 2007. Integración vertical en las empresas forestales comunitaria de Oaxaca. In Bray D, L Merino, D Barry eds. Los bosques comunitarios en México. México DF, México. INE-SEMARNAT. p. 303-324.

Bray D. 2007. El manejo comunitario de los bosques en México: veinte lecciones aprendidas y cuatro senderos para el futuro. In Bray D, L Merino, D Barry eds. Los bosques comunitarios en México. Cd. de México. INE-SEMARNAT. p. 417-434. 
Bray D, L Merino. 2005. La experiencia de las comunidades forestales en México. Veinticinco años de silvicultura y construcción de empresas forestales comunitarias. México DF, México. Secretaría de Medio Ambiente y Recursos Naturales, Instituto Nacional de Ecología, Consejo Civil Mexicano para la Silvicultura Sostenible A.C. y Fundación Ford. 272 p.

Blundell R, A Duncan, K Pendakur. 1998. Semiparametric estimation and consumer demand. Journal of Applied Econometrics 13: 435-46.

CONAPO (Consejo Nacional de Población, MX). 2012. Índice de marginación por localidad. Consultado $11 \mathrm{sep}$. 2016. Disponible en http://www.conapo.gob.mx/work/models/CONAPO/ indices_margina/2010/documentoprincipal/Capitulo01.pdf.

CONASAMI (Comisión Nacional de Salarios Mínimos, MX). 2016. Boletín de prensa de 11 de diciembre de 2005. Consultado 15 jul. 2016. Disponible en http://www.conasami. gob.mx/bol_salario_minimo_2016_11122015.html.

Cortés F, RM Rubalcava. 1993. Consideraciones sobre el uso de la estadística en las ciencias sociales. Estar a la moda o pensar un poco. In Méndez I, P González $\mathrm{C}$ eds. Matemáticas y Ciencias sociales. México DF, México. Miguel Ángel Porúa. p. 24-50.

Durán-Medina E, JF Mas, A Velázquez A. 2007. Cambios en las coberturas de vegetación y usos del suelo en regiones con manejo forestal comunitario y áreas naturales protegidas de México. In Bray D, L Merino, D Barry eds. Los bosques comunitarios en México. México DF, México. INE-SEMARNAT. p. 267-302

Fukuyama F. 2003. Capital social y desarrollo: la agenda venidera. In Atria R (CEPAL), M Siles (MSU), I Arriagada (CEPAL), LJ Robison (MSU), S Whiteford (MSU) eds. Capital social y reducción de la pobreza en América Latina y el Caribe, en busca de un nuevo paradigma. Santiago, Chile. Comisión Económica para América Latina y El Caribe/Michigan State University. p. 33-48.

Garibay C. 2005. El dilema corporativo del comunalismo forestal. Desacatos 23: 251-274.

Hardin G. 1968. The tragedy of the commons. Science 162: 1243:48

Infante S, GP Zárate de L. 2012. Métodos estadísticos: Un enfoque interdisciplinario. México DF, México. Colegio de Postgraduados. 609 p.

INEGI (Instituto Nacional de Estadística Geografía e Informática, MX). 2010. Censo de población y vivienda 2010. México. Consultado: 28 feb. 2014. Disponible en www.inegi.org.mx/ prod_serv/contenidos/espanol/.../AEGPEF_2013.pdf

Lambin E, BL Turner, HJ Geist, SB Agbola, A Angelsen, JW Bruce, OT Coomes, R Dirzo, GFischer, CFolke, PS George, K Homewood, J Imbernon, R Leemans, X Li, EF Moran, M Mortimore, PS Ramakrishnan, JF Richards, H Skånes, W Steffen, GD Stone, U Svedin, TA Veldkamp, C Vogel, J $\mathrm{Xu}$. (2001). The causes of land-use and land-cover change: moving beyond the myths. Global Environmental Change 11(4): 261-269. DOI: $\underline{10.1016 / \mathrm{S} 0959-3780(01) 00007-3}$

López B, A Velázquez, L Merino. 2010. Explorando los determi- nantes del buen manejo forestal comunitario. Interciencia 35 (8): 560-567.

Maignan I, OC Ferrell. 2004. Corporate social responsibility and marketing: an integrative framework. Journal of Academy of Marketing Science 32(1): 3-19. DOI: 10.1177/0092070303

Murillo D. 2004. Falacias de desarrollo sustentable: una crítica desde la metamorfosis conceptual. Economía, Sociedad y Territorio 4(16): 635-656.

Ostrom E. 2000. Social Capital: A fad or a fundamental concept? In P Dasgupta, I Serageldin eds. Social capital a multifaceted perspective. Washington, USA. The World Bank. 78 p.

Palacio-Prieto JL, G Bocco, A Velázquez, JF Mas, F Takaki, A Victoria, L Luna-González, G Gómez-Rodríguez, J LópezGarcía, I Trejo-Vázquez, A Peralta, J Prado-Molina, A Rodríguez-Aguilar, R Mayorga-Saucedo, F González. 2000. La condición actual de los recursos forestales en México: Resultados del Inventario Forestal Nacional 2000. Investigaciones Geográficas 43: 183-203.

PEF (Poder Ejecutivo Federal, MX). 2001. Programa de manejo de la Reserva de la Biosfera de la Mariposa Monarca. México (PDF). Consultado 27 nov. de 2018. Disponible en http://www.conanp.gob.mx/que hacemos/pdf/programas manejo/monarca.pdf

PNUD (Programa de las Naciones Unidas para el Desarrollo, US). 2014. Índice de desarrollo humano municipal en México: nueva metodología. Consultado 11 sept. 2016. Disponible en http://www.mx.undp.org/content/dam/mexico/docs/ Publicaciones/PublicacionesReduccionPobreza/InformesDesarrolloHumano/UNDP-MX-PovRed-IDHmunicipalMexico-032014.pdf

Porter M. 1991. The competitive advantage of nations. New York, USA. The Free Press. 320 p.

Prabhu R, CJP Colfer, P Venkatesawatan, LC Tan, R Soekmadi, E Wollenberg. 1996. Criteria and indicators for the sustainable man forests: Phase I final report. Bogor, Indonesia. CIFOR Special Publication. 230 p. DOI: 10.17528/cifor/000393

Rodríguez J, MJ González, E Valtierra. 2018. Análisis estratégico de la cadena productiva forestal en la región de la Reserva de la Biosfera de la Mariposa Monarca. Madera y Bosques 24(1): $20-36$

Simon H. 1979. Rational decision making in business organizations. American Economic Association 4(69): 493-513. DOI: $10.21829 / \mathrm{myb} .2018 .2411404$

Stonehouse G, B Snowdon. 2007. Competitive advantage revisited: Michael Porter on strategy and competitiveness. Journal of Management Inquiry 3(16): 256-273.

Torres-Rojo JM, A Guevara-Sanginés, DB Bray. 2007. La economía de la administración del manejo comunitario forestal en México: un estudio de caso en el Balcón, Tecpan, Guerrero. In Bray D, L Merino, D Barry eds. Los bosques comunitarios en México. México DF, México. INE-SEMARNAT. p. 341-374.

Velázquez A, JF Mas, JR Díaz-Gallegos, R Mayorga-Saucedo, PC Alcántara, R Castro, T Fernández, G Bocco, E Ezcurra, JL Palacio. 2002. Patrones y tasas de cambio de uso del suelo en México. Gaceta Ecológica 62: 21-37. 
